\title{
Análisis de la estrategia de RSC dirigida a la comunidad local. El caso de una multinacional
}

\section{Analysis of a multinational company CSR strategy for use with a local community}
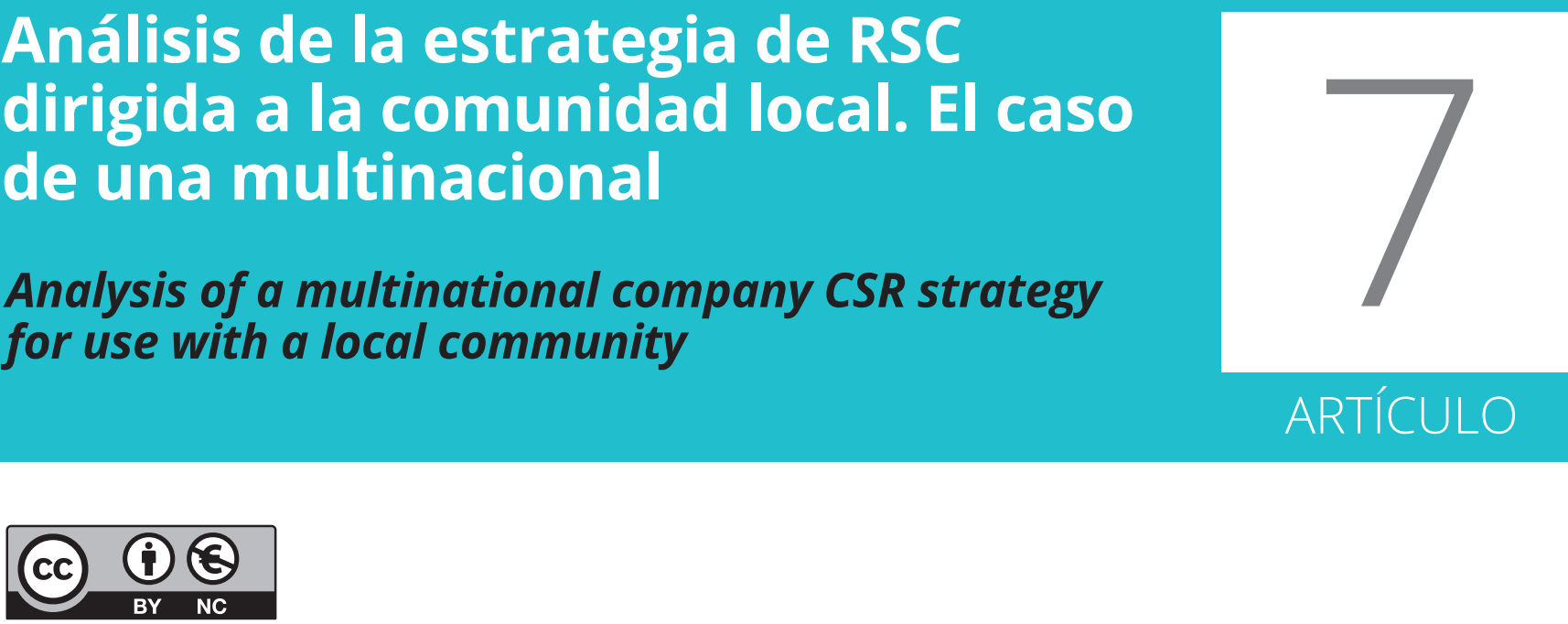

\section{Estrella Barrio Fraile}

Departamento de Publicidad, Relaciones Públicas y Comunicación Audiovisual Universitat Autònoma de Barcelona

Doctora en Publicidad y Relaciones Públicas por la Universitat Autònoma de Barcelona. Investigadora y profesora en la misma universidad. Miembro investigador del grupo Publiradio de la misma universidad. Es co-autora de capítulos de libros y artículos relacionados con la Responsabilidad Social Corporativa. También ha participado como ponente en diferentes congresos nacionales e internacionales.

estrella.barrio@uab.cat

ORCID: 0000-0001-8047-4393

\section{Ana María Enrique Jiménez}

Departamento de Publicidad, Relaciones Públicas y Comunicación Audiovisual Universitat Autònoma de Barcelona

Investigadora y profesora de la Universitat Autònoma de Barcelona y de la Universitat Abat Oliba donde imparte asignaturas de teorías de la comunicación y comunicación empresarial e institucional. Autora de diversos capítulos de libros y artículos relacionados con la comunicación de crisis y la Responsabilidad Social Corporativa. Coordinadora del grupo de investigación en Dirección de Comunicación Empresarial e Institucional (UAB).

anamaria.enrique@uab.cat

ORCID: 0000-0001-5902-403X

Fecha de recepción: 30 de noviembre de 2017 / Aceptación: 14 de marzo de 2018 


\section{Resumen}

Estamos asistiendo a un momento de cambio en el contexto empresarial, lo cual repercute directamente en el comportamiento de las organizaciones con sus stakeholders. En esta nueva realidad, analizamos, a partir de la metodología del estudio de caso, cómo la estrategia de Responsabilidad Social Corporativa (RSC) de una organización contribuye al desarrollo del stakeholder de la comunidad local. En síntesis, podemos concluir que las acciones de RSC dirigidas a la comunidad local que realiza la empresa analizada están alineadas con la estrategia global de RSC de la compañía.

\section{PALABRAS CLAVE}

Responsabilidad social, RSC, comunicación, estrategia, stakeholders, comunidad local.

\section{Abstract}

We are living through a period of great change in the business sector, which directly affects organisations' relations with stakeholders. In this new context, using a case study methodology, we have analysed how an organisation's Corporate Social Responsibility (CSR) strategy contributes to the relationship with local community stakeholders. We found that the CSR initiatives addressed to the local community by the company in the study are well-aligned with the company's global CSR strategy.

\section{KEYWORDS}

Social responsibility, CSR, communication, strategy, stakeholders, local community.

\section{INTRODUCCIÓN}

Estamos asistiendo a un momento de cambio en el contexto empresarial. En los últimos años han salido a la luz casos de ingeniería financiera, corrupción, violación de derechos laborales y desastres medioambientales que han llevado a la ciudadanía a desconfiar de las empresas. Afortunadamente, la sensibilidad social en el desarrollo empresarial y la preocupación de la ciudadanía por el deterioro medioambiental son cada vez mayores, hecho que, unido a la evolución de las Tecnologías de la Información y la Comunicación, ha provocado que las exigencias y expectativas de la sociedad en torno a las prácticas empresariales sean escuchadas. Las organizaciones han comprendido que no son un ente aislado, sino que forman parte de la sociedad y que su existencia a largo plazo depende de su legitimación ante ésta.
Por otro lado, no debemos olvidar que nos encontramos ante la sociedad del excedente, con empresas que rivalizan entre sí cuyos tangibles (productos, calidades, precios, garantías, etc.) son similares, por lo que la llave de la diferenciación entre las empresas y marcas se encuentra en la gestión de los valores intangibles. Costa (2012, p. 102), define esta situación actual como la de una sociedad en la que "cada día los valores éticos y de buen gobierno empresarial son más apreciados por los públicos y más exigidos por los stakeholders".

Ante esta nueva realidad empresarial protagonizada por el incremento de la desconfianza hacia las empresas y el auge de los valores intangibles, la Responsabilidad Social Corporativa (RSC) se presenta como un valor intangible clave para restablecer la confianza entre la empresa y sus públicos.

En este artículo nos interesa centrar el análisis de las estrategias de RSC de una compañía 
multinacional en el stakeholder de la comunidad local, ya que la articulación de este tipo de estrategias tiene un impacto directo en el desarrollo de ésta última debido a la posibilidad de interaccionar y obtener beneficios de manera recíproca.

\section{MARCO TEÓRICO}

\subsection{CONCEPTO DE RESPONSABILIDAD SOCIAL}

A lo largo de las últimas décadas, desde que se plantea, en 1956, la primera definición de RSC por parte de Bowen en su libro Social responsibilities of the businessman, son diversos los autores que han definido el concepto de RSC, y más diversas aún, son las definiciones que proponen. Sin embargo, se pueden atisbar una serie de criterios que parecen repetirse a la hora de explicar el concepto de RSC. Davis y Blosmstrom (1966), Walton (1967), Van Marrewijk (2003), Hopkins (2004), Basil y Weber (2006), Waldman et al. (2006), Castillo (2009) y Cuervo (2009) señalan la relación de la empresa con sus grupos de interés, mientras que autores como, Jones (1980), Cuesta y Valor (2003), Smith (2003), García-Marzá (2004) y Maignan y Ferrell (2004) hacen especial hincapié en un compromiso y/u obligación de la empresa hacia sus grupos de interés. Otras definiciones hacen referencia a aquellas acciones que realiza la empresa que van más allá de los intereses económicos de la organización (Davis, 1960; Mc Guire, 1963; Davis y Blomstrom, 1966; Eells y Walton, 1974), es decir, que buscan un bienestar socioeconómico general (Frederick, 1960; Kok et al., 2001). Otros autores destacan en sus definiciones la noción de integrar las preocupaciones sociales y medioambientales por parte de las empresas en el desarrollo de sus actividades de negocio (Cuesta y Valor, 2003;
Van Marrewijk, 2003; Panwar et al, 2006; Castillo, 2009; Cuesta y Valor, 2009; Cuervo 2009). Otro criterio que se repite a la hora de definir RSC, es la responsabilidad de las empresas por los impactos negativos en la sociedad derivados de sus actividades (Fitch, 1976; Mohr et al., 2001; Cuesta y Valor, 2003). En esta misma línea, otros autores destacan el deber de las empresas de contribuir al desarrollo y la mejora de la sociedad (World Business Council for Sustainable Development, 2000; Kok et al., 2001), maximizando la creación de valor compartido (Mohr et al., 2001; Comisión Europea, 2011).

En definitiva, podemos afirmar que la RSC es consecuencia de un compromiso adoptado con todos sus grupos de interés en materia económica, social y medioambiental, con el objetivo de responsabilizarse de las consecuencias e impactos que derivan de sus acciones y maximizar la creación de valor compartido para todo el conjunto de grupos de interés. La RSC no se queda en la superficie de las organizaciones, si no que va más allá de la propia acción social que implementen las empresas, no se trata de acciones puntuales y concretas de greenwashing. A diferencia de conceptos como la acción social, la filantropía o el marketing con causa, la RSC supone un planteamiento estratégico que afecta al conjunto de la organización y a todos sus públicos. La visión estratégica de la RSC implica que ésta se sitúa en lo más profundo de las compañías, trabajando siempre de acuerdo con la visión, misión y valores de la organización.

Una vez tratado el término de RSC, consideramos que puede resultar esclarecedor contextualizar la RSC en los principios que la inspiran. Para ello, se toma como referencia el documento Marco Conceptual de la Responsabilidad Social Corporativa de AECA (2004), dónde se presentan los seis principios básicos que constituyen las reglas fundamentales que rigen 
el comportamiento socialmente responsable de las empresas.

El primero de ellos es el principio de transparencia, que se refiere al acceso que tienen los grupos de interés a información sobre el comportamiento social de la organización, siendo la comunicación por parte de la empresa de los aspectos ligados a la RSC el instrumento esencial para cumplir este principio. El segundo es el principio de materialidad, que hace referencia al paso de la teoría a la práctica real de la empresa, teniendo en cuenta las necesidades y expectativas de los diferentes grupos de interés en la toma de decisiones. El tercero, el principio de verificabilidad, se basa en el sometimiento de las actuaciones de RSC a una verificación externa realizada por expertos independientes que comprueben la veracidad de las actuaciones socialmente responsables de la organización. El cuarto, denominado como el principio de visión amplia, está estrechamente vinculado con el concepto de sostenibilidad y la idea de responsabilizarse de las consecuencias e impactos que derivan de sus acciones. El principio de mejora continua está relacionado con la idea de una gestión continuada de la corporación, dado que el comportamiento socialmente responsable promueve la visión de largo plazo que aleja a la empresa de actividades puramente especulativas que sólo buscan la maximización de beneficios a corto plazo, sin considerar sus consecuencias en el largo plazo. Y por último, el principio de naturaleza social de la organización supone que las estrategias de RSC procuren que la organización se estructure en base a su naturaleza social y no sobre valores de índole estrictamente técnico-económica.

Merece especial atención el principio de visión amplia, que hace hincapié en la visión microeconómica y macroeconómica de la organización. Citando a AECA:
La organización debe centrar sus objetivos de responsabilidad social corporativa en el contexto de sostenibilidad más amplio posible. Debe considerar el impacto que produce a nivel local, regional, continental y global, con un sentido claro de legado para futuras generaciones. (AECA, 2004, p. 21)

Este principio supone tener en cuenta, a la hora de aplicar políticas de RSC, a todo el conjunto de grupos de interés de la organización, ya sea a nivel global como puede ser la sociedad en general o los proveedores que una compañía tiene repartidos por todo el globo terráqueo, o a nivel mucho más específico y regional, como puede ser la comunidad local.

\subsection{DE LA TEORÍA DE LOS STAKEHOLDERS A LA COMUNIDAD LOCAL}

No se puede hablar de RSC, sin antes comprender el nuevo paradigma de empresa, que ha cambiado la forma de entender las organizaciones, estando formadas por un conjunto de stakeholders con objetivos e intereses dispares por los que la empresa debe velar.

Es importante, aclarar el significado y origen de esta palabra, stakeholder, que ha sido traducida al castellano como "participante" (Perdiguero, 2003) o grupo de interés (Villafañe, 2004). El término stakeholder, representa un juego de palabras cuyo origen de partida sería el vocablo shareholder o stockholder (accionista), el que posee una acción o participación. Así, siguiendo este juego de palabras, de la unión de los vocablos stake (interés) y holder (poseedor), nace el término stakeholder, que no define otra cosa, que a aquel que posee un interés con la empresa, definido por Freeman (1984, p. 25) como "cualquier grupo o individuo que 
pueda afectar o ser afectado por el logro de los objetivos de la empresa."

Por consiguiente, hemos pasado a un nuevo modelo de empresa complejo, definido por los equilibrios y relaciones entre la compañía y los stakeholders. En palabras de Perdiguero, este nuevo enfoque propone:

Una visión de la empresa mucho más compleja que la establecida en la teoría económica neoclásica, asignando a la dirección la obligación de gestionar en función de las necesidades, expectativas e intereses de todos los grupos e individuos afectados por sus actividades. (Perdiguero, 2003, p. 158).

En definitiva, no se puede entender la RSC sin comprender este nuevo paradigma de empresa formada por todo el conjunto de grupos de interés que la componen, de los cuales depende su propia supervivencia, motivo por el cual el objetivo económico de la empresa consiste en crear valor para todo el conjunto de stakeholders, satisfaciendo las necesidades de cada uno de ellos.

El siguiente paso sería preguntarse cuáles son los diferentes grupos de interés, que conforman el mapa de stakeholders de una organización. Si bien cada empresa tendrá sus propios participantes específicos que dependerán de cada industria, existen una serie de stakeholders principales que de manera genérica abordan todas las organizaciones. En este sentido, destacan los trabajos de Svendsen (1998), Freeman, Harrison y Wicks (2008), Comisión Europea (2001) y AECA (2007) que proponen y clasifican los diferentes stakeholders (Figura 1).

Es interesante observar que para este conjunto de autores e instituciones, no existe una clasificación y definición unánime de los diferentes stakeholders que componen el mapa de públi-
Primarios: Accionistas e inversores, consumidores, empleados, proveedores, competidores y socios industriales

Secundarios: Comunidad, medioambiente, especies no humanas y generaciones futuras, medios de co-

municación y gobiernos y entes reguladores

(Svendsen, 1998)

Primarios: Clientes, empleados, proveedores, financieros y comunidades

Secundarios: Gobierno, competencia, grupos de los consumidores, medios de comunicación y grupos de especial interés

Freeman, Harrison y Wicks (2008)

Dimensión interna: Incluye las prácticas responsables en lo social (empleados) y las prácticas respetuosas con el medio ambiente dentro de la empresa

Dimensión externa: Comunidades locales, socios comerciales, proveedores, consumidores, derechos humanos, problemas ecológicos mundiales (Comisión Europea, 2001)

Partícipes primarios, contractuales o directos: Internos: Accionistas y empleados

Externos: Clientes, proveedores, acreedores financieros, socios comerciales y comunidad (cuando ésta invierte en infraestructuras de utilidad para la empresa)

Partícipes complementarios, contextuales o indirectos: Comunidades locales o territoriales, sociedad, público en general, generaciones futuras, competidores, Administración Pública, agentes o interlocutores sociales, ONG's y medios de comunicación. (AECA, 2007)

Figura 1. Clasificación de stakeholders. Fuente: Elaboración propia

cos de una organización no obstante existen cinco grupos de interés, que bajo diferentes nomenclaturas, están presentes en todas las propuestas. Estos son: accionistas, consumidores, empleados, proveedores y comunidad.

De especial interés es el stakeholder de la comunidad local, y es que tal y como afirma la Comisión Europea (2001, p. 12) la RSC "abarca también la integración de las empresas en su entorno local [...] las empresas contribuyen al desarrollo de las comunidades en que se insertan, sobre todo en las comunidades loca- 
les". Por su parte, Svendsen (1998) señala que la comunidad está formada por los residentes que viven en un área cercana a la empresa, asociaciones de vecinos, cámaras de comercio, ONG's, escuelas y universidades y grupos de especial interés. Sub-categorías que van en la línea de la definición propuesta por AECA (2004, p. 15) que define la comunidad local como el "conjunto de entidades de iniciativa pública o privada del entorno local circundante a la actividad desarrollada por la empresa, con el que se interactúa estrechamente".

Finalmente, en relación a las propias acciones de RSC que una compañía puede llevar a cabo en la comunidad local, la Comisión Europea, las define del siguiente modo:

Muchas empresas se comprometen con la sociedad local a través de, por ejemplo, el ofrecimiento de plazas adicionales de formación profesional, la colaboración con organizaciones de defensa del medio ambiente, la contratación de personas socialmente excluidas, el ofrecimiento de servicios de guardería a sus trabajadores, el establecimiento de asociaciones con comunidades, el patrocinio de actividades deportivas o culturales a nivel local o la realización de donaciones para obras de beneficencia. (Comisión Europea, 2001, p. 13)

\section{METOdOLOGÍA}

En la presente investigación se ha abordado concretamente la articulación de la estrategia de RSC en el stakeholder de la comunidad local. Para ello, se ha planteado una investigación basada en el método del estudio de caso. Este método de investigación es muy apropiado cuando el objetivo es comprender y explicar un fenómeno utilizando las fuentes de información que se requieran. En esta investigación no pretendemos buscar una representación estadística. Nuestra aspiración es alcanzar una generalización analítica en la medida en que como explica Coller (2005, p. 68) "el caso es pertinente teóricamente (es decir, relevante), se puede relacionar las conclusiones del estudio con una teoría o conjunto de ellas [...]". Estas conclusiones no se refieren a una población o universo, sino a unas teorías previas.

La casuística analizada ha sido la multinacional Unilever. La elección de esta compañía como caso de estudio viene determinada por el criterio de las investigadoras que consideran a priori que éste puede ser un caso que permite simbolizar las teorías presentadas en el marco teórico, debido a su manera de entender la RSC, ya que cuenta con un plan global de RSC y un sub-plan dirigido a la comunidad local, denominado Plan Unile»er Cirnparte. Se trata una compañía relevante en los sectores de la alimentación, el cuidado del hogar y la higiene personal, con una amplia gama de productos que se comercializan en este país. La empresa cuenta con un amplio reconocimiento a su RSC. En 2014 lideró rankings como el Tomorrow Value's Rating y la encuesta GlobeScan/ Sustainability y fue galardonada con los premios CSR Online Awards, el Premio Nacional de Marketing a la Ética y la Sostenibilidad y el Gothenburg Award for SustainAbility Development. También está presente en los medios de comunicación especializados en RSC como ejemplo de buenas prácticas, como son las publicaciones Corresponsables, Compromiso RSE y Diario Responsable.

El diseño de la investigación, se ha basado en la triangulación que como señala Soler (2011, p.192) consiste en "contrastar la información [...] la acción de reunión y cruce dialéctico de toda la información pertinente al objeto de es- 
tudio surgida en una investigación". Por tanto se ha combinado la revisión de documentos escritos, con la realización de entrevistas enfocadas a actores que forman parte de la implementación del Plan Unilever Comparte y observaciones de distintas acciones de RSC dirigidas a la comunidad que lleva a cabo la compañía:

- Revisión de tres documentos escritos.

- Documento Plan Unilever para una Vida Sostenible.

- Documento Plan Unilever Comparte: Guía de desarrollo de la comunidad.

- Web corporativa de Unilever España.

- Realización de cuatro entrevistas enfocadas a los siguientes agentes informantes.

- Ana Palencia. Directora de Comunicación y RSC de Unilever España. Miembro del Comité de Dirección de Unilever España. Lidera la comunicación europea de Unilever en materia de sostenibilidad.

- Imma Pérez. Directora de la consultora de RSC y comunicación Nottopic. Colaboradora externa del Departamento de Comunicación de Unilever España.

- Marina Jarque. Directora de Comunicación Corporativa en el Ayuntamiento de Viladecans.

- Miguel Vallejo. Responsable de Servicios a Empresas del Ayuntamiento de Viladecans.

- Realización de cinco observaciones a los siguientes eventos.

- Taller RSE en su dimensión social: la empresa y su relación con el territorio programado dentro de la 63 jornada Corresponsables.

- Actividad Desayuno saludable «pan con Tulipán» e higiene bucal Signal.
- Acto de clausura del concurso El Gran Estalvi.

- Actividad Taller de autoestima Dove.

- Acción Caminata Solidaria.

Los ejes temáticos que han guiado la recolección de datos y la clasificación de la información obtenida son:

- Plan estratégico internacional de RSC de Unilever.

- Plan Unilever Comparte: Acciones de RSC dirigidas a la comunidad local.

La recogida de datos se llevó a cabo entre los meses de febrero y junio de 2015. Para la recogida de datos a través de las entrevistas enfocadas, se elaboró un guión esquemático con los ítems conceptuales a tratar con cada uno de los agentes informantes. En el caso de las observaciones, se creó un fichero de catalogación con las categorías a tener en cuenta en cada una de las observaciones.

Posteriormente, el análisis de datos se realizó en dos etapas. Una primera etapa de clasificación de la información en función de los ítems conceptuales fijados en el guión de la entrevista. Una segunda etapa, posterior al análisis de contenido de las entrevistas y observaciones realizadas, en el que se codificó el contenido en función de las categorías de análisis establecidas.

\section{RESULTADOS}

A continuación se presentan los resultados definitivos de la investigación en función los ejes temáticos previamente establecidos. 


\subsection{PLAN ESTRATÉGICO INTERNACIONAL DE RSC DE UNILEVER}

Unilever cuenta con un plan estratégico de RSC a diez años (desde 2010 hasta el año 2020) que engloba a los 190 países en los que opera la multinacional. Este plan se denomina Plan Unilever para una Vida Sostenible.

El lanzamiento de este plan tiene su origen en el cambio de presidente de la empresa. En el año 2009 se incorpora a Unilever el actual presidente mundial, Paul Polman, quien marca la nueva estrategia de la compañía orientada a desarrollar nuevas formas de hacer negocio con el objetivo de duplicar el tamaño de la compañía, al mismo tiempo que se reduce el impacto medio ambiental y se incrementa de manera positiva el impacto social.

A partir de ese momento Paul Polman, decide poner orden en las diferentes acciones de RSC que ya realizaba la empresa y lanza, en 2010, el Plan Unilever para una Vida Sostenible, asentado en tres pilares fundamentales: salud y bienestar, medioambiente y calidad de vida. Este plan establece tres objetivos generales para el año 2020, que se basan en los tres pilares fundamentales. Estos son:

- Pilar de salud y bienestar, cuyo objetivo es ayudar a más de mil millones de personas a mejorar sus hábitos en salud y bienestar.

- Pilar de medioambiente, cuyo objetivo es reducir a la mitad el impacto medioambiental provocado por la fabricación y el uso de sus productos.

- Pilar de calidad de vida, cuyo objetivo es mejorar la calidad de vida de cientos de miles de personas en la cadena de suministro.

Estos tres objetivos generales están compuestos por diferentes objetivos específicos, que a su vez, se desglosan en sub-objetivos más concretos, que se plasman en las diferentes acciones de RSC que lleva a cabo esta multinacional a nivel global.

\subsection{PLAN UNILEVER COMPARTE - ACCIONES DE RSC DIRIGIDAS A LA COMUNIDAD LOCAL}

El Plan Unilever Comparte, cuyo objetivo es crear un impacto positivo en la comunidad en la que opera la compañía, es un proyecto que nace en 2012 para trabajar una dimensión más cercana y concreta del Plan Unilever para una Vida Sostenible. Por consiguiente, se trata de un plan de RSC a pequeña escala que reúne un conjunto de acciones dirigidas al municipio de Viladecans (Barcelona), donde se encuentra la sede de Unilever España. Dichas acciones se encuentran alineadas con los tres pilares del plan global de RSC de la empresa.

A continuación, se describen las diferentes acciones que conforman el Plan Unilever Comparte, clasificadas en función de los tres pilares que constituyen el Plan Unilever para una Vida Sostenible.

Pilar de salud y bienestar:

- Desayuno saludable "pan con Tulipán" e higiene bucal Signal. Se trata de talleres sobre hábitos alimentarios dirigidos a los alumnos de primero y segundo de primaria de las escuelas de Viladecans, cuyo objetivo es transmitir a los niños las pautas para una correcta alimentación e higiene bucal. Estos talleres se ofrecen a los centros educativos del municipio a través del Área de Educación del ayuntamiento, tienen una duración aproximada de una hora y veinte minutos y se realizan en los propios centros educativos en horario escolar con la presencia de los/ las maestros/as en el aula. Las charlas son 
impartidas por nutricionistas contratados/as y formados/as por el Área de Nutrición y Salud de Unilever para la ocasión. El taller en sí consta de:

- Una explicación de la importancia que tiene realizar un desayuno saludable, que debe incluir cuatro tipos de alimentos: cereales o farináceos, lácteos, fruta y grasas saludables. Y una explicación de cómo debe ser un correcto lavado de los dientes.

- Tres juegos en los que los niños ponen en práctica lo explicado anteriormente.

- La realización de un desayuno saludable en el aula con alimentos aportados por la compañía. Este desayuno contiene un vaso de leche con cacao, una rebanada de pan de molde con margarina Tulipán (producto de Unilever), dos galletas Flora (producto de Unilever) y una brocheta de fruta.

- Talleres de Autoestima Dove. Esta actividad, que se realiza bajo el paraguas de la marca producto Dove de Unilever, consiste en la realización de charlas dirigidas a jóvenes de primero y segundo de la ESO de Viladecans con el objetivo de cambiar la visión que tienen de su propia imagen y hacerles ganar más confianza apostando por una belleza real. Estas charlas se ofrecen a los centros educativos del municipio a través del Área de Educación del ayuntamiento, tienen una duración aproximada de una hora y se realizan en los propios centros educativos en horario escolar con la presencia de los/las profesores/as en el aula. Los talleres son impartidos por voluntarios de diferentes departamentos de la compañía. La actividad en sí consta de:
- Una explicación que incluye un recorrido histórico de los ideales de belleza, el origen de la presión de los ideales de belleza, cómo los medios manipulan las imágenes creando cuerpos irreales y los problemas que causa esta presión y manipulación, tratando de manera especial el papel que juegan las redes sociales como elementos de presión social en el entorno de los jóvenes.

- Un juego en parejas en el que los alumnos deben decir qué les gusta de su compañero/a.

- Un debate entre los alumnos sobre lo aprendido y trabajado durante la sesión.

Pilar de medioambiente:

- Concurso Canciones de 5 minutos para la ducha. Con el objetivo de fomentar la reducción del consumo de agua, la compañía y el ayuntamiento diseñan un concurso en el que se invita a las escuelas del municipio a idear una canción creativa de cinco minutos para el momento del baño que trate sobre el buen uso del agua. Con esta acción se pretende diseminar la idea de duchas de cinco minutos. Al tratarse de centros educativos, la convocatoria para participar se realiza a través del ayuntamiento. La escuela ganadora recibe material para el centro.

- Concurso El Gran Estalvi. Esta iniciativa, que consiste en un concurso dirigido a los vecinos y comunidades de vecinos del municipio para ver quién reduce más su consumo de agua, luz y gas durante un periodo de cuatro meses, tiene como objetivo concienciar a la población en el ahorro del agua y la energía. Cuando una persona o comunidad de vecinos se apunta para participar en el concur- 
so, recibe un pack formado por un manual de instrucciones para ahorrar energía, una bombilla led y un reloj de arena con una ventosa para ponerlo en el baño y controlar que el tiempo de la ducha no supera los cinco minutos. Durante los cuatro meses que dura el concurso, los participantes deben mirar el contador dos o tres veces e ir entregando sus facturas. Al finalizar el concurso, los ganadores reciben un pack de productos Unilever y una dotación económica, que va entre los $200 €$ y los 6000€, dependiendo de la categoría en la que participen, para invertir en seguir mejorando la eficiencia energética de las viviendas. La creación y diseño de este concurso se realizó entre Unilever y el ayuntamiento de Viladecans (Áreas de Infraestructuras y Medioambiente). La ejecución se lleva a cabo entre la multinacional y el ayuntamiento, con el apoyo de un clúster local de empresas sobre eficiencia energética.

Pilar de calidad de vida:

- Caminata Solidaria. Es una marcha solidaria cuya finalidad es recaudar fondos para la Fundación Viladecans Solidaria que ayuda en el pago de necesidades básicas, como alimentación, medicamentos o luz, a familias de la ciudad que lo necesiten. Tiene un recorrido de $5 \mathrm{~km}$ que cruza el municipio de Viladecans, se organiza anualmente el primer fin de semana de junio y es apto para todos los públicos. Los participantes pagan una inscripción de $4 €$ y a cambio reciben una camiseta para la caminata y un pack de productos Unilever que se entrega al final del recorrido, momento en el que también se venden productos de la empresa cuya recaudación también se destina a la fundación. Esta acción se lleva a cabo entre Unilever, el ayuntamiento, el colegio San Gabriel y el básquet femenino de Viladecans.
- Reparto de alimentos y productos de necesidad básica. La compañía colabora con Cáritas, Cruz Roja y los servicios sociales del ayuntamiento en el reparto de alimentos y productos de necesidad básica a familias del municipio por debajo del umbral de la pobreza.

- Cursos de cocina. El chef de Unilever realiza cursos de cocina en las instalaciones que la compañía tiene en el municipio para enseñar a elaborar menús de manera responsable y económica a familias de Viladecans desestructuradas y/o con pocos recursos, con la finalidad de ayudarles a aprender a comer de manera equilibrada utilizando pocos recursos. Estos cursos son organizados por la empresa con la colaboración de los servicios sociales del ayuntamiento.

- Colaboración con CAVIGA y ASDIVI. La compañía colabora con CAVIGA (un centro ocupacional para personas con disminución psíquica de Viladecans) y ASDIVI (una asociación para la integración de personas con discapacidad de Viladecans) realizando diferentes actividades de voluntariado, como por ejemplo almuerzos, para los usuarios de estos centros.

- Conferencias a pymes. Se trata de charlas que realizan directivos de la multinacional para compartir conocimiento con pequeñas y medianas empresas del territorio. A modo de ejemplo, se pueden mencionar las conferencias realizadas por Yolanda Menal y Ana Palencia, directoras de RRHH y Comunicación de Unilever España, sobre cómo fomentar la motivación y sobre cómo mejorar la comunicación interna. Además de compartir conocimiento, estos encuentros también promueven el networking y la relación entre Unilever y las empresas del territorio, ya que al acabar el acto, estos altos cargos de la 
compañía mantienen conversaciones en petit comité con los asistentes.

- Market Place. Unilever participa en estos encuentros entre pymes y grandes empresas, denominado Market Place, que organiza el Área de Servicios a Empresas del Ayuntamiento de Viladecans. Esta actividad consiste en una especie de evento de networking vinculado a unos retos específicos, marcados para cada edición por el ayuntamiento, en el que la gente, empresarios y consistorio se conocen y cuya finalidad es establecer relaciones de confianza entre los participantes.

- Coaching a jóvenes en riesgo de exclusión social. Unilever colabora con el programa Proyecto Coach de la Fundación Exit a través de coaching a jóvenes de entre 15 y 17 años que no han acabado la ESO y están realizando un curso de formación profesional. La Fundación Exit realiza unos programas de cualificación profesional inicial para jóvenes no graduados en educación secundaria obligatoria. Dentro de estos programas, unos 15 o 20 voluntarios de la empresa hacen de coach de uno de estos jóvenes. Durante tres meses se reúnen cada viernes con ellos para orientarles, animarles y ayudarles. Durante estos tres meses, cada viernes de 9h a 14h acuden a las oficinas de la compañía y se les enseñan los diferentes departamentos, cómo trabajan, qué realizan, etc.

- Programa Voluntariado UNIdos en Viladecans. Además de las actividades de voluntariado con CAVIGA, ASDIVI y la Fundación Exit, Unilever cuenta con un programa de voluntariado interno, según el cual, cada empleado puede dedicar ocho horas de voluntariado anuales dentro de su jornada laboral para la realización de actividades solidarias. Con el objetivo de afianzar la relación de la compañía con la comunidad local, muchas de esas actividades se realizan el municipio de Viladecans, como son la entrega de rosas en un centro de día para mayores el día de Sant Jordi, cuenta cuentos a niños hospitalizados - la retirada de plantas invasoras de la playa de la localidad.

\section{DISCUSIÓN Y CONCLUSIONES}

Los resultados de la investigación muestran que Unilever cuenta con un plan estratégico de RSC a 10 años asentado en tres pilares fundamentales que marcan los tres objetivos generales a cumplir en el 2020. Estos son: salud y bienestar, medioambiente y calidad de vida.

A su vez, la compañía cuenta con un plan denominado Plan Unilever Comparte que consiste en la adaptación del plan global de RSC de la organización al stakeholder de la comunidad local, el municipio de Viladecans, donde se encuentra la sede de Unilever España. Las acciones de RSC dirigidas a la comunidad que realiza la empresa para cada uno de los tres objetivos generales de su plan global de RSC son:

- Pilar de salud y bienestar: Desayuno saludable "pan con Tulipán" y Talleres de Autoestima Dove.

- Pilar de medioambiente: Concurso Canciones de 5 minutos para la ducha y concurso El Gran Estalvi.

- Pilar de calidad de vida: Caminata Solidaria, reparto de alimentos y productos de necesidad básica, cursos de cocina, colaboración con CAVIGA y ASDIVI, conferencias a pymes, participación en el Market Place, coaching a jóvenes en riesgo de exclusión social y programa de voluntariado UNIdos en Viladecans. 
En definitiva, a través de esta investigación se ha conseguido ejemplificar cómo una organización multinacional articula la estrategia de RSC en el stakeholder de la comunidad. Gracias a la presente investigación, sabemos que las acciones de RSC dirigidas a la comunidad local que realiza la empresa analizada están alineadas con la estrategia global de RSC de la compañía. No estamos ante acciones puntuales aisladas, sino que se trata de un sub-plan que se deprende de la estrategia general de RSC de la organización.

Por otra parte, esta investigación también nos ha permitido conocer que el stakeholder de la comunidad local se segmenta en diversos sub-grupos de interés. Estos sub-grupos se clasifican en dos categorías: los stakeholders beneficiarios y los partners. Los primeros hacen referencia a aquellos a los que la compañía dirige sus acciones de RSC, mientras que los segundos son los que colaboran con la empresa en la articulación del programa de RSC en la comunidad local. En el caso del Plan Unilever Comparte, aquellos sub-grupos a los que la empresa dirige sus acciones de RSC están formados por: población juvenil, familias por debajo del umbral de la pobreza, familias desestructuradas, pymes y jóvenes en riesgo de exclusión social, entre otros. En cuanto a los partners, éstos incluyen el Ayuntamiento de Viladecans de manera genérica y algunas áreas en concreto como Educación, Medioambiente, Servicios Sociales o la Fundación Viladecans Solidaria y diferentes agentes sociales como los centros educativos del municipio, el básquet femenino de Viladecans y las ONG's Cruz Roja, Caritas, Caviga, Asdivi y Fundación Exit.
Podemos concluir que este caso analizado es un ejemplo de cómo es posible por parte de las empresas ir más allá de la implementación de acción social, es decir, que es posible por parte de las organizaciones articular estrategias de RSC que se sitúan en lo más profundo de su gestión, que entienden la RSC como una oportunidad para establecer un compromiso real en materia económica, social y medioambiental que pueda beneficiar a su entorno, en este caso a la comunidad, y por ende, maximizar de este modo, la creación de valor compartido. 


\section{BIBLIOGRAFÍA}

AECA (2007). Gobierno y Responsabilidad Social de la Empresa. Madrid: Asociación Española de Contabilidad y Administración de Empresas.

AECA (2004). Marco Conceptual de la Responsabilidad Social Corporativa. Madrid: Asociación Española de Contabilidad y Administración de Empresas.

Basil, D.Z. y Weber, D. (2006). Values motivation and concern for appearances: the effect of personality traits on responses to corporate social responsibility. International Journal of Nonprofit and Voluntary Sector Marketing, 11(1), 61-72.

Castillo, A. (2009). Relaciones Públicas. Teoría e historia. Barcelona: Editorial UOC.

Coller, X. (2005). Estudio de casos. Madrid: Centro de Investigaciones Sociológicas.

Comisión Europea. (2011). Estrategia renovada de la UE para 2011-2014 sobre la responsabilidad social de las empresas. Bruselas: Comisión Europea.

Comisión Europea. (2001). Libro Verde de la Comisión Europea para Fomentar un Marco Europeo para la Responsabilidad Social de las Empresas. Bruselas: Comisión Europea.

Costa, J. (2012). El DirCom hoy. Granollers (Barcelona): CPC Editor.

Cuervo, A. (2009). Responsabilidad social corporativa, gobierno de la empresa y stakeholders. En J. J. Almagro., J. A. Garmendia e I. de la Torre. (Coords.), Responsabilidad social: una reflexión global sobre la RSE (p. 51-61). Madrid: Pretince Hall.

Cuesta, M. y Valor, C. (2009). La Responsabilidad social empresarial vista por las organizaciones sociales. En J. J. Almagro., J. A. Garmendia e I. de la Torre. (Coords.), Responsabilidad social: una reflexión global sobre la RSE (p. 135-146). Madrid: Pretince Hall.

Cuesta, M. y Valor, C. (2003). Responsabilidad social de la empresa: concepto, medición y desarrollo en España. Boletín Económico De ICE, Información Comercial Española, (2755), 7-20.

Davis, K. y Blomstrom, R.L. (1966). Business and its environment. New York: McGrawHill. 
Davis, K. (1960). Can business afford to ignore social responsibilities? California Management Review, 2(3), 70-76.

Eells, R. y Walton, C. (1974). Conceptual foundations of business. Burr Ridge, II: Irwin.

Fitch, H. G. (1976). Achieving corporate social responsibility. Academy of Managemnt Review, 1, 38-46.

Frederick, W.C. (1960). The growing concern over business responsibility. California Management Review, 2(4), 54-61.

Freeman, R. E., Harrison, J. S. y Wicks, A. C. (2008). Managing for Stakeholders: Survival, Reputation, and Success. New Haven, Connecticut: Yale University Press.

Freeman, R. E. (1984). Strategic Management. A Stakeholder Approach. Boston: Pitman.

García-Marzá, D. (2004). Ética empresarial. Del diálogo a la confianza. Madrid: Trotta.

Hopkins, M. (2004). The Planetary Bargain. Corporate Social Responsibility Matters. Londres: Routledge.

Jones, T.M. (1980). Corporate social responsibility revisited, redefined. California Management Review, 22(2), 59-67.

Kok, P., van der Wiele, T., McKenna, R. y Brown, A. (2001). A corporate social responsibility audit within a quality management framework. Journal of Business Ethics, 31(4), 285-297.

Maignan, I. y Ferrell, O.C. (2004). Corporate social responsibility and marketing: An integrative framework. Journal of the Academy of Marketing Science, 32(1), 3-19.

McGuire, J.W. (1963), Business and society. New York, NY: McGraw-Hill.

Mohr, L. A., Webb, D. J. y Harris, K. E. (2001). Do consumers expect companies to be socially responsible? The impact of corporate social responsibility on buying behavior. The Journal of Consumer Affairs, 35(1), 45-72.

Panwar, R., Rinne, T., Hansen, E. y Juslin, H. (2006). Corporate responsibility: Balancing economic, environmental, and social issues in the forest products industry. Forest Products Journal, 56(2), 4-12.

Perdiguero, T.G. (2003). La responsabilidad social de las empresas en un mundo global. Barcelona: Anagrama. 
Smith, N. C. (2003). Corporate social responsibility: Not whether, but how? Centre for Marketing, 03-701, 1-37.

Soler, P. (2011). La investigación cualitativa. Un enfoque integrador. En L. Vilches. (Coord.), La investigación en comunicación. Métodos y técnicas en la era digital (p. 189-236). Barcelona: Gedisa.

Svendsen, A. (1998). The stakeholder strategy. San Francisco, California: Berrett-Koehler.

Van Marrewijk, M. (2003). Concepts and definitions of CSR and corporate sustainability: Between agency and communion. Journal of Business Ethics, 44(2/3), 95-105.

Villafañe, J. (2004). La buena reputación. Madrid: Pirámide.

Waldman, D.A., de Luque, M.S., Washburn, N. y House, R.J. (2006). Cultural and leadership predictors of corporate social responsibility values of top management: A GLOBE study of 15 countries. Journal of International Business Studies, 37(6), 823-837.

Walton, C.C. (1967). Corporate social responsibilities. Belmont, CA: Wadsworth.

World Business Council for Sustainable Development, (2000). Corporate social responsibility: making good business sense. Londres: WBCSD. 